\title{
Understanding artwork
}

\author{
Given that an article can't be published until any artwork issues are resolved, it makes sense to get it \\ right first time and avoid delays.
}

When giving seminars about publishing in Nature Photonics, one question that invariably comes up is about artwork and what's expected from authors. Given the frequency of the question, and that around $30 \%$ of all the artwork we receive is initially unsuitable for printing, it seems prudent to clarify matters and provide guidance on how to avoid any potential pitfalls.

Artwork is generally supplied to Nature Photonics for one of three reasons: either it is material for use in a primary research paper (Letter or Article) or in a general-interest section of the journal (News and Views, Commentaries, Interviews, Review Articles and so on), or it is a cover suggestion for an issue containing an author's research paper.

Artwork for primary research papers is usually uploaded through our web-based manuscript submission system, although we do have a dedicated file-transfer protocol (FTP) system in place that can be used for particularly large files. An editor can provide login details on request.

In contrast, artwork for the cover or for use in the general-interest section of the journal is usually sent to the relevant editor by e-mail (although again FTP can be used if necessary). In the case of commissioned general-interest articles, we are also able to assist authors by redrawing any suggestions for illustrations - please just send a suitable sketch that is clear enough for our art editor to professionally reproduce.

When supplying artwork, it is important to understand that it can be categorized into one of the following four types: (1) 'photographic' image data that has been generated from a digital camera or imaging device and is inherently composed of pixels; (2) charts and graphs that show experimental data or simulations; (3) schematics and diagrams; and (4) multimedia files such as short movies for the supplementary information of research papers.

Here's how each should be handled. Most importantly, charts, graphs and schematics must be supplied as 'vector' format files (that is, they are not resolution-dependent). 'Vector' here refers to the lines and points that make up the figure and its individual elements. This in turn highlights the other key aspect of vector graphics: they remain editable, allowing our art editor to restyle the figure without altering or redrawing

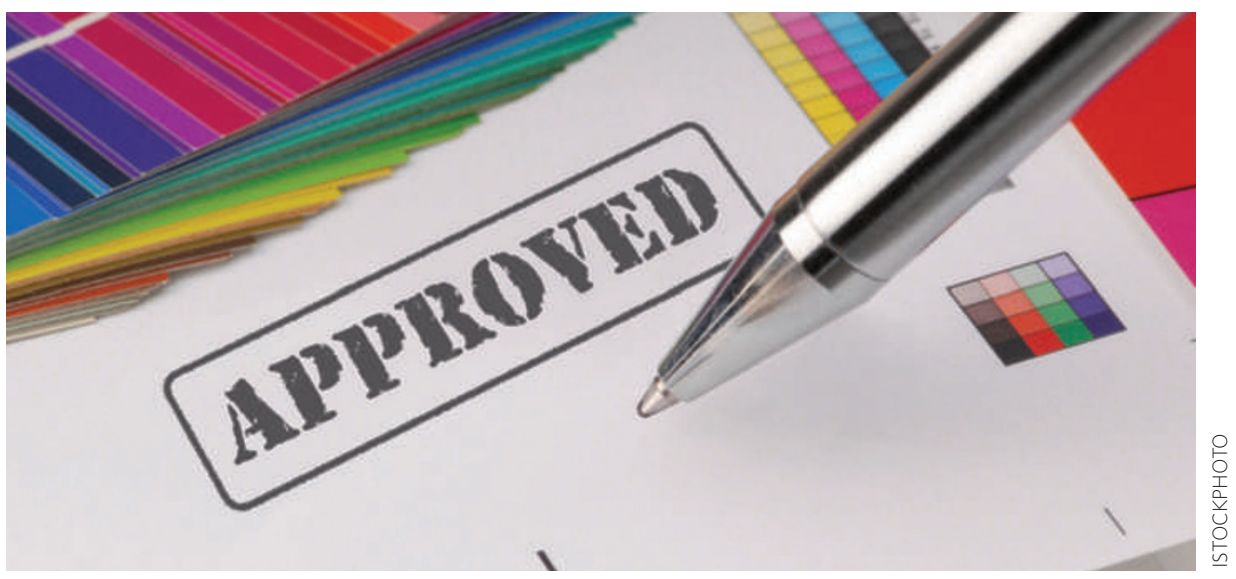

any of the actual data. Compatible formats are EPS, AI or PDF files. In all cases please ensure that they are saved in an editable (unflattened) format so that labels, arrows and other parts can be adjusted to meet out style requirements. These options can usually be found in the 'save as' or 'export' options of most popular graphics software packages.

Pixel-based image data should ideally be supplied in its raw, unmanipulated state, without any annotations, at a resolution close to 300 dots per inch, and as a TIFF, JPEG or BMP file. This effectively means at least 700 pixels is required for a single-column figure. Please feel free to send images even if their resolution is slightly lower than this (due to hardware limitations, for example), but be aware that they may be unsuitable for printing if their quality is too poor. If the images have been manipulated in any way, it is important to explain how and why they have been processed.

As for user-generated charts and graphs, the most important issue is to maintain clarity and avoid ambiguity. Please be sure to label both axes along with units and, if possible, provide errors bars together with an explanation of their origin. For clarity it is usually good practice to distinguish separate data sets with different colours and to avoid squeezing too many curves onto a single graph. With schematics and diagrams, the issue of clarity is again paramount. Please ensure that every part of the figure is clearly labelled, and that any acronyms used are defined in the either the main text of the article or the figure caption. In all cases please ensure that captions are succinct and do not exceed 150 words.

Movie and audio files accompanying a research paper should be uploaded via our manuscript submission system as supplementary information files with a brief explanation of their contents. Files in most common formats and up to several megabytes in size are acceptable.

When sending cover suggestions please also provide the relevant manuscript number to avoid any potential confusion. The best cover images are always strong, eye-catching visual statements that portray the key concept of their associated research, rather than intricate and complex technical drawings. Our art editor is able to take designs and refine/redraw them if you do not have sophisticated graphics software at your disposal.

Finally, it is important to mention copyright and image permissions. If you are sending a piece of artwork that you have not generated yourself (that is, it belongs to someone else or has been reproduced elsewhere) please be sure to either obtain reprint permission from the source publisher or, if you are unsure how to do this, contact the Nature Photonics editor in question who will provide further guidance. In the case of Review Articles, which usually contain a large number of previously published figures, please be sure to complete the accompanying figure permissions form, which will provide us with the necessary source information for each figure (full journal citation details) and so allow appropriate permissions to be obtained. 